\title{
Riconoscere la criminalità d'impresa: il caso Eternit di Casale Monferrato
}

\author{
Rebecca Paraciani
}

\section{Riassunto}

Vi sono dei crimini, gli whitecollarcrime, che faticano ad essere percepiti come tali. Nonostante il loro potenziale danno sociale ed economico sia elevato, è come se venissero declassati, considerati "meno reati"e, dunque, puniti in maniera diversa. Caratteristiche strutturali li rendono meno percepibili e più tollerati, tanto che il whitecollar sarà più facilmente definito "disonesto", piuttosto che "criminale". Questo articolo racconta la ridefinizione dell'evento dannoso avvenuta a Casale Monferrato, ospite di uno degli stabilimenti della società Eternit. Comincia da qui una guerra all'amianto che, dall'interno della fabbrica, arriva alla cittadinanza non immune alle scelte dettate dalla ricerca del mero profitto. Scioperi, lotte, petizioni porteranno questa cittadina ad ottenere la messa al bando dell'amianto nel 1992 e attireranno l'attenzione del PM Guariniello, che coglie l'impolverato filo rosso che collega i diversi stabilimenti facenti capo al gruppo Eternit, dando vita al"processo del secolo", tristemente caduto in prescrizione il 19 Novembre 2014.

\section{Résumé}

Certains crimes, en particuliers ceux qui sont commis par les cols blancs, sont rarement perçus comme des comportements criminels. Bien que la possibilité de dommage social et économique soit élevée, il semble qu'ils soient déclassés, considérés « moins criminels » que d'autres types de crimes, et donc sanctionnés différemment.

Des caractéristiques structurelles les font qu'on les perçoit moins et qu'on les tolère mieux que d'autres crimes, à tel point qu'un criminel en col blanc sera plus facilement défini comme une personne malhonnête que comme un criminel.

Cet article présente la redéfinition du fait dommageable qui s'est passé à Casale Monferrato (Italie) où se trouvait l'une des usines Eternit. La "guerre » à l'amiante commence ici et, de l'intérieur de l'usine, se répand parmi la population qui n'est pas insensible aux choix exclusivement dictés par les profits.

\section{Abstract}

Some crimes, in particular white-collar crimes, are rarely and with many difficulties perceived as crimes. Despite the known potential social and economic damage they cause, it seems as though they were declassified, considered "less criminal" than other kinds of crimes, and therefore punished in a different way. Many specific characteristics make them less perceived and easier to tolerate than other crimes, so much that a white collar perpetrator will be easily defined as a "dishonest" person rather than a "criminal".

This article presents the redefinition of the harmful event occurred in Casale Monferrato (Italy) where one of the Eternit plants was located. The "asbestos war" begins here starting from inside the factory spreading to citizens who are not indifferent to choices which are exclusively dictated by profit motive.

Key words: white-collar crimes; Eternit; Casale Monferrato; asbestos war; citizens.

\section{Eternit, dal latino aeternitas, a sottolineare l'eterna durevolezza e resistenza del materiale. Ma l'eternità ha un prezzo.}

\section{Introduzione.}

A Casale Monferrato lo stabilimento Eternit è "l'America": un lavoro sicuro, una paga sicura e anche buona, orari che consentono ai lavoratori di coniugare lavoro e cura delle proprie terre. La Eternit è la materializzazione del progresso.

Ma a che prezzo? Qual è il senso del lavorare se lo si fa per morire?

\footnotetext{
- Dottore in "Scienze criminologiche per l'investigazione e la sicurezza". Si occupa di progettazione e ricerca sociale a favore dell'inclusione, della sicurezza, delle pari opportunità e dei diritti umani, per contrastare qualsiasi forma di discriminazione e promuovere la giustizia sociale.
} 
Questa storia, che ben rappresenta le criticità attinenti al rapporto tra lavoro e salute, vede protagonisti non solamente i lavoratori, ma l'intera cittadinanza. Le modalità di lavorazione e gestione dell'amianto, materiale tanto resistente quanto dannoso, erano tali da non garantire la tutela dei lavoratori e neanche quella della comunità di Casale Monferrato. Il polverino era ovunque. Gli scarti di produzione, poi, venivano regalati dal generoso stabilimento: Eternit nelle strade, Eternit nei campi da calcio delle parrocchie, Eternit come isolante sui tetti, Eternit ovunque. A Casale Monferrato si respirava Eternit, che significa ammalarsi, "prendere la polvere", contrarre tipologie tumorali letali che possono insorgere fino a quarant'anni dopo l'esposizione al materiale dannoso. Il lungo periodo di latenza della malattia, in primo luogo, rende poco chiara la correlazione diretta tra il comportamento nocivo dello stabilimento e il danno per la popolazione; inoltre, pone nel mirino dell'amianto-killer chi oggi è nel pieno delle proprie forze ed è stato esposto quando l'Eternit era ancora attiva, cioè fino al 1986, come i bambini che correvano sul polverino nel proprio giardino, coloro che venivano allattati da una madre-lavoratrice dello stabilimento, nella pausa, con ancora addosso l'impolverata tuta che prima del turno era blu, chi oggi è un adulto con una famiglia e un lavoro.

Qual era il prezzo di questo "posto sicuro"? E chi lo sta pagando?

Tutta la comunità casalese è direttamente coinvolta e colpita. Oltre al rischio di ammalarsi, vi è la paura di ammalarsi. Se sei di Casale Monferrato vivi con una spada di Damocle sulla testa, consapevole che oggi si è ammalato qualcuno e che ci sarà un prossimo e poi un altro e un altro ancora.

Ma Casale Monferrato reagisce. Una rivolta dopo l'altra, un obiettivo dopo l'altro, fino ad arrivare a richiedere un riconoscimento processuale dell'ingiustizia, costruendo, grazie al PM Raffaele Guariniello e alla sua équipe, quello che, per più elementi, verrà definito lo "storico processo", tristemente finito in prescrizione il 19 Novembre 2014.

\section{Tra teoria e giurisprudenza.}

"Tutti $i$ cittadini banno pari dignità sociale e sono eguali davanti alla legge 1 ", recita il primo comma dell'articolo 3 della nostra Costituzione; in base a questo principio di fronte alla legge dovrebbero annullarsi tutte le differenze etniche, politiche, economiche e sociali. Non sempre, però, questo accade.

Già nel 1939 Sutherland denunciò come, nonostante dalle statistiche ufficiali emergesse una forte correlazione tra criminalità e condizione socio-economica svantaggiosa, in realtà vi fossero dei reati, quelli dei colletti bianchi, che non venivano considerati dalle statistiche e non venivano perseguiti penalmente. Rientrano nel concetto di "crimine del colletto bianco" quei delitti commessi da un soggetto rispettabile, di elevata condizione sociale, nell'ambito della propria occupazione, attività o professione, con l'abuso della fiducia che deriva proprio dalla posizione socio-economica, nonché dalla professione svolta o dal ruolo ricoperto ${ }^{2}$.

\footnotetext{
${ }^{1}$ Cost. Italiana, «Principi fondamentali», art. 3, c. I.

2 Bravo F., "Criminalità economica violenta, compilancemodels e rating di legalità delle imprese", in Balloni A., Bisi R., Sette R. (a cura di),
} 
Questi illeciti non soltanto causano danni economici di gran lunga superiori a quelli generati dalla criminalità delle "persone comuni", ma rappresentano anche una grave minaccia all'ordine morale ${ }^{3}$. Nonostante questo, vengono puniti con una pena solamente come extrema ratio, quasi a voler tutelare il responsabile dall'etichetta di "criminale". La responsabilità principale di questa differenza di trattamento è del legislatore, "o meglio, dell'ipocrisia delle pratiche di attuazione della legge4", che dimostrano chiaramente che il reato altro non è che una costruzione sociale che rispecchia il volere della classe dominante.

L'insistenza sul concetto di reato serve a mettere in evidenza come la posizione di rilievo dei colletti bianchi venga spesso utilizzata per sottrarre la condotta deviante di questi ultimi alla sanzione penale, collocandola piuttosto in sede civile o amministrativa, quasi depenalizzando azioni che, in realtà, nel momento in cui le si comparano con i delitti disciplinati dal Codice Penale, possono anche risultare più gravi e dannose. Questa depenalizzazione, unita alla capacità di questi soggetti "rispettabili” di potersi permettere una difesa legale brillante e di poter utilizzare a proprio vantaggio conoscenze influenti, va a destigmatizzare ${ }^{5}$ le proprie condotte, che non vengono percepite né come

Manuale di criminologia Volume II, Bologna, Clueb, 2013, pp. 39-86.

${ }^{3}$ Cottino A., Disonesto ma non Criminale. La giustizia e i privilegi di potenti, Roma, Carrocci, 2005.

${ }^{4}$ Cottino A., Ibidem, p. 21.

5 Goffman nel 1964, nella sua opera Stigma. L'identità negata, analizza le pratiche di inferiorizzazione sociale. Il soggetto, nel momento in cui è portatore di uno stigma, subisce un declassamento, divenendo oggetto di biasimo. Lo stigma definisce una diversità declinata in termini di inferiorità. devianti, né tantomeno come criminali da parte della collettività. Il diritto penale viene applicato in modo da evitare che lo stigma del crimine colpisca il reo-colletto bianco ${ }^{6}$ per diversi motivi. Innanzitutto sono loro tra $i$ primi a non riconoscersi devianti e spesso agiscono come guidati da un "sentimento di impunità" . Manca, poi, un meccanismo di denuncia adeguato, dovuto alla difficoltà nel rintracciare un nesso causale, diretto e immediato, tra autore e vittima del reato. Spesso, inoltre, è proprio la posizione "altolocata" dei white collar a consentire loro di ripulirsi facilmente dallo stigma, sia perché li aiuta nell'ottenimento di cariche istituzionali prestigiose, in grado di far dimenticare l'illecito commesso, sia perché hanno la possibilità di servirsi dei media a proprio favore ed evitando un ammonimento morale nei confronti di questi illeciti "a-penali"s. Attraverso un linguaggio utilizzato con astuzia e parole scelte con attenzione (e intenzione), "ci viene sottratta la possibilità di vedere la natura violenta di un atto, di una situazione, di un processo in corso; o, se preferiamo, ci viene data l'opportunità di non vederli"’; sarà più facile che a queste condotte venga attribuita l'etichetta di "disoneste", "scorrette", piuttosto che quella di "criminali".

Altro fattore che contribuisce a ridimensionare la portata di tali condotte è la proliferazione di false credenze, in primis l'assenza di conseguenze per altre persone coinvolte. Se si considerano gravi crimini di impresa, come per esempio lo scandalo di Tangentopoli, a rimetterci non sono

\footnotetext{
${ }^{6}$ Sutherland E. H., Il crimine dei colletti bianchi. La versione integrale, Milano, Giuffrè editore, 1987.

${ }^{7}$ Martucci P., La criminalità economica. Una guida per capire, Roma-Bari, Laterza, 2006.

${ }^{8}$ Sutherland E. H., Op. Cit.
} 
state direttamente le grandi imprese, bensì i contribuenti: "siamo infatti stati sicuramente noi cittadini a pagare la somma di 1328 miliardi di lire spesi per la metropolitana di Napoli, contro i 49 inizialmente previsti"10.

Altra falsa credenza è la convinzione che non si tratti di una criminalità violenta. Anche questa da sfatare. Consideriamo la definizione di violenza fornita dalla World Health Organization che, nel World Report on violence and health del 2002, definisce violenta qualsiasi condotta che, attraverso l'abuso di potere o di forza fisica, minaccioso o reale, contro una persona o un gruppo di persone o una comunità, risulta o ha un'alta probabilità di risultare in lesione fisica, morte, danno psicologico, non sviluppo o deprivazione ${ }^{11}$. Questa definizione si sposa con la criminalità di impresa: basta infatti sostituire ad "abuso di potere o di forza fisica" la formula “abuso di potere economico". Si conferma, così, sia il carattere violento della criminalità di impresa, sia la sua elevata dannosità sociale ${ }^{12}$.

Nel nostro ordinamento giuridico la responsabilità delle imprese è regolata da una normativa ad hoc, il decreto legislativo 231/2001, che disciplina la responsabilità amministrativa delle persone giuridiche, delle società e delle associazioni, anche prive di personalità giuridica. La normativa traduce una serie di provvedimenti internazionali che hanno manifestato l'esigenza di contrastare la criminalità economica, rendendo l'impresa protagonista di interventi atti

\footnotetext{
${ }^{9}$ Cottino A., Op. Cit., p. 78.

${ }^{10}$ Cottino A., Ibidem, p. 92.

$11 \mathrm{WHO}$, World report on violence and health, Geneva, 2002.

12 Bravo F., "Criminalità economica violenta, compilancemodels e rating di legalità delle imprese", in Balloni A., Bisi R., Sette R. (a cura di), Op. Cit.
}

a tutelare l'interesse generale ${ }^{13}$. L'obiettivo per il quale tale decreto nasce è quello di ridurre il rischio di reato. A seguito di questo provvedimento, la corretta gestione dell'azienda non si valuta più affidandosi soltanto al risultato economico che essa ottiene, ma anche ad elementi come la responsabilità etica, sociale ed ambientale. Il decreto legislativo 231/2001, in base a quanto enunciato nel primo articolo, ha introdotto uno specifico sistema punitivo destinato agli enti, scardinando lo storico principio secondo cui societas delinquere non potest e rendendo soggetti punibili gli enti forniti di personalità giuridica, le società e le associazioni anche prive di personalità giuridica ${ }^{14}$. Il principio di responsabilità delle imprese è derivante dalla commissione di uno degli illeciti facenti parte dell'elenco dei reati-presupposto, da parte di soggetti agenti in nome o per conto dell'ente. Questo nuovo tipo di responsabilità si affianca a quella penale individuale $\mathrm{e}$, in questo modo, l'ente non risponde più solamente in via sussidiaria, ma anche in via esclusiva. È solo dal 2011 che, all'interno dell'elenco dei reatipresupposto, sono entrate a far parte alcune fattispecie di reati ambientali, ma ne sono state escluse altre, molto importanti, tra cui il reato di inquinamento atmosferico, quello di avvelenamento delle acque e, ancora, quello di disastro innominato ambientale ${ }^{15}$. Sebbene sia positivo il riconoscimento dellimpresa quale

\footnotetext{
${ }^{13}$ Galluccio L., Gavino P., Responsabilità penale e amministrativa delle imprese, Gruppo 24Ore, Milano, 2009.

${ }^{14}$ Il criterio seguito è quello secondo il quale la posizione dell'ente è equiparata a quella della persona fisica.

15 AssOnime, Reati ambientali e responsabilità amministrativa dell'ente, Diritto societario, Circolare n. 15, 28 Maggio 2012.
} 
attore giuridico, rimangono alcune perplessità sull'efficacia di questi provvedimenti. Innanzitutto, per far sì che l'ente possa essere considerato responsabile è necessario dimostrare che il reato-presupposto sia stato commesso a suo interesse o a suo vantaggio: si tratta di un passaggio molto difficile, anche a causa del tecnicismo di alcune tipologie di reato, presupposto dunque che permette di escludere un cospicuo numero di delitti imprenditoriali. Alle imprese viene, poi, fornito un importante salvagente: la possibilità di implementare ex post un modello organizzativo, di risarcire il danno, di rimuovere le conseguenze dannose della propria condotta. Questo attenua le sanzioni pecuniarie ed elimina l'afflizione di quelle interdittive, più efficaci e idonee ad imprimere nell'immaginario collettivo la definizione di "impresa criminale". Infine non va sottovalutata l'esitazione con la quale la magistratura utilizza gli strumenti sanzionatori previsti dal decreto: è esiguo il numero dei provvedimenti e manca un quantitativo rilevante di condotte illecite.

Opportuno soffermarsi sulla reazione di Confindustria riguardo l'estensione della responsabilità amministrativa delle società ai reati ambientali: sostiene l'eccessiva ampiezza dell'elenco dei reati-presupposto, vorrebbe l'esclusione delle sanzioni interdittive in relazione ai reati ambientali e critica il fatto che, nella valutazione dei modelli organizzativi delle imprese, non venga considerata l'adozione di certificazioni volontarie ambientali ISO 14001 e EMAS $^{16}$.

\footnotetext{
${ }^{16}$ Si tratta di attestati che certificano l'impegno di un'organizzazione per il rispetto dell'ambiente. L'impresa sceglie volontariamente di ottenere questa certificazione, che viene conferita solo in presenza di un Sistema di Gestione Ambientale.
}

Definire "eccessivamente ampio" un elenco di reati presupposto può essere letto con perplessità: sarebbe come se qualcuno sostenesse troppo ampio l'elenco dei reati previsti dal Codice Penale. Penso. È proprio uno dei punti di forza del D. Lgs. 231/2001 il suo essere in continuo aggiornamento, il suo riuscire a comprendere, a poco a poco, i reati che potrebbero danneggiare dal singolo cittadino all'intera comunità. Comprensibile, invece, la critica al mancato riconoscimento delle certificazioni volontarie ambientali ISO 14001 e EMAS nella valutazione del modello di organizzazione. Il fatto che l'impresa implementi un sistema di gestione ambientale (SGA) è sicuramente segnale dell'introduzione di un sistema generale di organizzazione, pianificazione delle attività, definizione di responsabilità, prassi e procedure che consentono all'impresa di agire in maniera rispettosa dell'ambiente, a favore di un'attiva politica ambientale. Non considerare tali certificazioni nella valutazione del modello organizzativo previsto dal D. Lgs. 231/2001 potrebbe essere sintomo di una mancanza di fiducia nei confronti di questi provvedimenti, evidentemente importanti (almeno a livello teorico) per la diffusione di una sensibilità ambientale dell'impresa. Partendo dal presupposto che tali certificazioni non possano considerarsi né sostitutive dei modelli organizzativi né, tantomeno, essere il presupposto per una presunzione di idoneità, si ritiene possano essere reputate una valida base di riferimento.

Ovviamente tali certificazioni consentono di avere tariffe agevolate. 
Gli illeciti imprenditoriali riescono, ancora oggi, ad evitare le conseguenze che investirebbero i cittadini comuni. Potrebbe essere una soluzione quella proposta da alcuni di introdurre tra i reati presupposto previsti dal D. Lgs. 231/2001 i reati intesi nel più ampio senso criminologico definito da Sutherland, estendendo l'ambito di applicazione anche agli illeciti a-penali, ma pur sempre di elevata dannosità sociale ${ }^{17}$.

\section{Casale Monferrato e lo stabilimento}

\section{Eternit $^{18}$.}

L'amianto è un minerale costituito da fibre sottilissime. In natura se ne possono trovare diverse varietà, appartenenti a due categorie principali: l'amianto di serpentino e l'amianto di anfibolo $^{19}$.

La loro struttura è costituita da fibre sottili e molto addensate che conferiscono a questo minerale alta resistenza ed estrema flessibilità; inoltre esse presentano anche le proprietà dell'assorbimento acustico, dell'isolamento termico e sono resistenti ad usura ed abrasione. È comprensibile, allora, il motivo per cui questo materiale sia presente in innumerevoli costruzioni e prodotti con i quali ci confrontiamo quotidianamente.

Nel 1902 Ludwig Hatschek brevettò il cementoamianto, meglio noto come eternit, che consiste nella commistione di acqua, cemento e fibre di

17 Bravo F., "Criminalità economica 'violenta', compilance models e rating di legalità delle imprese", in Balloni A., Bisi R., Sette R., Op. Cit.

${ }^{18}$ I dettagli della storia dello stabilimento Eternit a Casale Monferrato mi sono stati raccontati da Bruno Pesce, Nicola Pondrano e Romana Blasotti durante le rispettive interviste che ho condotto a Casale Monferrato il 24 Settembre 2014 e il 27 Gennaio 2015.

${ }^{19}$ Voce "Amianto", Enciclopedia Treccani Vol I, p. 293. amianto, facile da laminare in forma di lastre piane o ondulate, o trafilate per la costruzione di tubi. Questo brevetto fu il punto di partenza di società indipendenti in Belgio, Inghilterra e Svizzera; in Italia, il 6 Gennaio 1906, l'ingegnere Adolfo Mazza avviò la produzione di cementoamianto, fondando la "Società Anonima EternitPietra Artificiale".Casale Monferrato si trova, a partire dal 19 Marzo 1907, ad ospitare lo stabilimento più grande di proprietà dell'ingegnere Mazza; la cittadina viene scelta per la sua strategica posizione al centro del triangolo commerciale Genova-Torino-Milano. La fabbrica, estesa su 94.000 metri quadrati, viene costituita nell'area industriale del Ronzone, area di circa 200.000 metri quadrati; l'Eternit ne occupa il $47 \% \%^{20}$. Si tratta di 94.000 metri quadrati di opportunità occupazionali. Lo stabilimento casalese altro non è che la materializzazione del futuro, del progresso, con i suoi vantaggi, ma, soprattutto, con i suoi svantaggi.

Il ciclo produttivo della fabbrica era tale da non curarsi minimamente della salute dei lavoratori e delle lavoratrici che ve ne prendevano parte. In diversi reparti l'amianto veniva lavorato senza protezioni, eccetto, in alcuni casi, inutili mascherine di carta; se i macchinari si bloccavano, i lavoratori dovevano spurgarli, a mani nude. Tra le lavorazioni più dannose, quelle a secco, che generavano una costante e presente nube di amianto. Uno dei reparti più impolverati era quello dello stoccaggio, sommerso di polvere bianca e in diretta comunicazione con l'esterno: facile immaginare come gli agenti atmosferici

\footnotetext{
${ }^{20}$ Altopiedi R., Un caso di criminalità di impresa. L'Eternit di Casale Monferrato, L'Harmattan Italia, Torino, 2011.
} 
abbiano potuto disperdere con facilità la polvere oltre le mura dello stabilimento. Allo stesso modo, a disperdere tra le strade di Casale Monferrato gli effetti nocivi dell'amianto sono state le modalità di trasporto di tale materiale: caricato su camion scoperti che attraversavano la città, oppure trasportato dai cosiddetti "uominibicicletta" su di un carrello, inutile dirlo, senza protezione alcuna. Solamente negli ultimi anni di produttività dello stabilimento sono state prese alcune minime precauzioni, più per mettere a tacere i primi dubbi sulla nocività del materiale da parte dell'opinione pubblica, piuttosto che per reale interesse per la salute dei lavoratori. Tra questi metodi vi erano delle ventoline che aspiravano le polveri di tornitura e che avrebbero dovuto filtrarle per renderne possibile il riciclo, rilasciando nell'ambiente aria pulita; per il corretto funzionamento sarebbe stata necessaria una manutenzione quotidiana, a scapito della settimanale decisa dalla dirigenza. L'effettiva modalità di gestione delle "ventoline" lasciava che le polveri di tornitura venissero disperse nell'atmosfera cittadina, in balia dei venti, senza considerare, poi, la polvere che fuoriusciva dal portone del reparto a pressione, che comunicava con l'esterno e veniva lasciato aperto durante l'estate e, in inverno, aperto ogni quarto d'ora per la circolazione dei camion.

Le condizioni di lavoro alla Eternit erano nocive non solo per la salute fisica, ma anche per quella mentale. Dietro alla nube di polvere si celava una politica paternalistica per la quale un intervento limitatore della libertà di una persona, come appunto essere nel posto di lavoro per un determinato lasso di tempo, era attuato nell'interesse della persona stessa, che doveva quindi essere grata al datore di lavoro e dunque non poteva sottrarsi alle regole di condotta imposte.

Nell'esperienza casalese, i presunti benefattori mettevano in atto sfruttamento oltre misura (ad esempio implementando diverse lavorazioni a cottimo) e vere e proprie forme di ricatto. Nei rapporti lavorativi con i superiori vi era una netta separazione tra élite istruita e colta, che gestiva ogni tassello della società, e la massa lavoratrice, ignorante e succube, che poteva contare solamente sulla propria forza e sul proprio lavoro.

Da considerare, anche, come l'azienda fosse pienamente al corrente dei rischi amiantocorrelati. A confermare questo vi sono diversi studi, che risalgono agli anni Quaranta, ma i cui risultati sono stati censurati dal cartello europeo del cemento-amianto, nato per annullare la concorrenza in una condizione di oligopolio. 亡̀ nel 1954 che il dottor Doll, dopo aver notato una ricorrenza di cancro polmonare 10 volte maggiore nei lavoratori a contatto con l'amianto rispetto alla popolazione non esposta, si rifiutò di occultare gli esiti. L'anno successivo pubblicò sul British Journal of Industrial Medicine gli sconcertanti risultati, compiendo un passo verso la consapevolezza dei rischi legati alla lavorazione del materiale.

È opportuno fare una precisazione: sono gli anni del dopoguerra e la ricostruzione che li caratterizza sembra non poter procedere senza amianto che, infatti, registrerà, sino agli anni Settanta, un'impennata nei consumi. In questi stessi anni, le fabbriche adottano misure minime per evitare la comparsa dell'asbestosi. Questa tecnica viene definita da Carnevale "cronicizzazione": una minima attenzione in più garantisce una più lunga sopravvivenza dei 
lavoratori a contatto con l'eternit, la patologia appare più tardi e più lentamente progredisce, destando uno scandalo inferiore nell'opinione pubblica $^{21}$.

A testimonianza della conoscenza dei rischi amianto-correlati da parte dell'azienda, il fatto che i lavoratori delle zone più pericolose avevano lo stipendio incrementato di circa ventimila lire rispetto agli altri per l'“indennità di polvere".

Questi, però, sono anche gli anni durante i quali i lavoratori cominciano a notare che l'elenco, attaccato all'ingresso dell'Eternit, dei nomi dei colleghi che hanno perso la vita è sempre più lungo. Lavorando all'Eternit si "prendeva la polvere". Ammalarsi di polvere era vissuto quasi come un'amara tragica consolazione, come una medaglia al valore dopo anni di duro lavoro e grandi sacrifici. Inoltre era considerato normale. Per decenni, infatti, nonostante le morti per cancri professionali o asbestosi siano tuttora più del triplo rispetto alle morti per lavoro, erano morti silenti, ovvia conseguenza di un duro lavoro. Ma i lavoratori non ci stanno. Per fermare le malelingue che raccontavano che di amianto si poteva morire, i dirigenti mettevano in atto ricatti e minacce, specialmente contro $\mathrm{i}$ lavoratori dalle voci più sonore, ai quali, per esempio, eliminavano l'indennità di polvere. Nicola Pondrano, all'epoca giovane sindacalista, racconta come per prima cosa, visto che a due mesi dall'assunzione faceva già parte del consiglio di fabbrica dell'Eternit, bloccarono la sua carriera di tecnico di laboratorio (motivo per il quale era andato alla Eternit) affidandogli

${ }^{21}$ Carnevale F. e Chellin E., Amianto. Miracoli, virtù, vizi, Firenze, Tosca, 1992. mansioni diverse. L'azienda, inoltre, erogava un litro d'olio d'oliva al mese per ogni lavoratore. Veniva più volte detto, ai lavoratori: "se non siete bravi vi togliamo l'olio". Altra ritorsione messa in atto nei confronti dei lavoratori non innocui era quella di isolarli fisicamente dal resto degli operai, collocandoli in un reparto soprannominato "il Cremlino". Questo non era un luogo fisico, ma una condizione punitiva di lavoro duro, usurante e umiliante, che andava dalla pulizia delle latrine alla pulizia dei filtri delle polveri. Spesso i turni punitivi erano durante le ore notturne, quando la stanchezza era sempre in agguato e c'era il rischio di cadere nei contenitori da otto metri di altezza. Alla Eternit il lavoro era svolto sotto la pressione costante dei capi squadra, spesso ex operai, che avevano il timore di perdere il minimo comando che era stato loro dato. Altra pratica di ricatto un po' più velata e insidiosa era quella di spostare i lavoratori che manifestavano l'intenzione di licenziarsi in reparti meno nocivi, più "confortevoli", allo scopo di revocare il diritto del lavoratore all'indennità di rendita di passaggio 22 .

Tutte queste situazioni avvelenavano l'ambiente lavorativo al pari dell'amianto, sottoponendo i lavoratori ad uno stress psicologico che li paralizzava, sia per gli aspetti connessi al contesto lavorativo, sia per quelli riguardanti il contenuto del lavoro: relazioni scadenti con la dirigenza, ricatto, isolamento fisico (e conseguentemente sociale), orari duri, eccessivo carico di lavoro, sono tutte variabili che vanno a ridurre la qualità dell'ambiente lavorativo.

\footnotetext{
${ }^{22}$ La rendita di passaggio è erogata dall'INAIL ai lavoratori che contraggono, nel corso della propria attività lavorativa, la silicosi o l'asbestosi.
} 
Con l'interesse crescente per la salute nei luoghi di lavoro da parte di milioni di lavoratori, anche Casale Monferrato diviene teatro di importanti scioperi e storiche conquiste per ottenere miglioramenti a tutela della salute dei lavoratori.

Nel 1976 viene istituita la Commissione ambientale del consiglio di fabbrica, che decise di effettuare indagini ambientali per verificare la concentrazione di fibre d'amianto con propri periti. Emerse che in tutti i reparti di produzione erano presenti quantità notevoli di fibre e ciò contribuì ad incrementare la presa di coscienza della nocività del proprio lavoro tra gli operai Eternit che, autonomamente, cominciano a cercare informazioni sui rischi per la propria salute. Lo stabilimento reagì con minime (e insufficienti) precauzioni e con campagne di contro-informazione. Le testate dei giornali locali esultavano: “All'Eternit l'amianto non nuoce più"23. Ancora, l'azienda imponeva ai lavoratori di non fumare con campagne che ammonivano "fumare fa male", per limitare il numero di malati: infatti fumare accelerava la corrosione dell'organismo da parte dell'amianto. Lo scopo di questi slogan era quello di relativizzare la portata delle informazioni da parte degli oppositori all'amianto che nuoce alla salute, sì, ma solo se si fuma, o solamente se lavorato non in sicurezza. I lavoratori si sono mobilitati, vogliono Giustizia e le loro azioni, che partono da scioperi e arrivano sino alle aule del tribunale, catalizzano l'attenzione della stampa locale e dei concittadini.

Nel frattempo a Zurigo viene deciso di lasciar fallire la società: non solo per la crisi del mercato del cemento-amianto, ma anche per il

\footnotetext{
${ }^{23}$ Altopiedi R., Op. cit., p 56.
}

disinteresse del gruppo svizzero di investire in materiale alternativo. Il numero di morti tra $i$ lavoratori aumenta. La preoccupazione è crescente e la verità sta venendo a galla.

Il 4 Giugno 1986 lo stabilimento Eternit di Casale Monferrato è dichiarato fallito. L'azienda pare voler riprendere l'attività nel 1987, ma un'ordinanza emessa dal sindaco Riccardo Coppo, che vieta su tutto il territorio casalese l'impiego di amianto, lo impedisce.

Si comincia a capire che di amianto si può morire anche se non si è lavorato alla Eternit, ma la battaglia non viene immediatamente appoggiata dall'intera comunità.

"Eravamo disfattisti, sabotatori, gente che va in giro a dire baggianate, che vuole farsi vedere, creare casini ... è colpa vostra se è chiusa la Eternit, complimenti! Potevamo ancora averla, bel disastro che avete combinato".

Queste le parole di Bruno Pesce 24 mentre mi racconta le reazioni da parte dell'opinione pubblica. L'accusa che veniva mossa era quella di aver tolto da Casale l'America, il posto sicuro, come se il disastro non fosse costituito dalle migliaia di morti che la Eternit continuava a lasciare dietro di sé. Inoltre a non far comprendere subito la reale entità del rischio era la campagna di disinformazione messa in atto dalla Eternit stessa, che negava. Passava dall'affermare l'inesistenza del rischio al sostenere la sua avvenuta risoluzione. Ma la Eternit era la Eternit.

\footnotetext{
${ }^{24}$ Bruno Pesce è uno dei custodi della memoria storica della reazione di Casale Monferrato. Ha supportato i lavoratori fin dagli inizi di questa lotta, come sindacalista. E' ora coordinatore dell'Afeva, associazione dei familiari delle vittime dell'amianto.
} 
"Loro si vestivano molto meglio di noi, avevano le scarpe lucide e questo conta", continua Bruno Pesce. In fin dei conti "lo diceva l'Eternit". Il pregiudizio di classe andava a sminuire questa lotta per una presa di posizione partitica, mentre lo spirito della lotta era politico, della polis, dunque appartenente alla dimensione della vita comune, allo Stato e al cittadino. Era una lotta di diritti e dignità, non una lotta di partiti.

La pretura di Casale avviò un'indagine tesa ad accertare la responsabilità di tali morti, per verificare se la causa fosse della mancata implementazione di misure di cautela all'interno della fabbrica. La fase istruttoria durò cinque anni, dal 1985 al 1990, e si concluse con il rinvio a giudizio dei quindici dirigenti della società italiana. I capi di accusa sono omicidio colposo plurimo e lesioni colpose plurime aggravate, che coinvolgono legali rappresentanti, direttori generale e di stabilimento. Il processo si conclude nel 1993 con la condanna di quattro dei quindici imputati ${ }^{25}$. Durante gli anni del processo, con la legge 257 del 1992, la norma casalese di messa al bando dell'amianto viene estesa a tutta Italia, paese che per primo in Europa, con le regole relative alla cessazione dell'impiego dell'amianto, impedisce l'attività di estrazione, lavoro ed impiego della fibra killer.

E' il 1988, due anni dopo il fallimento della Eternit, data di fondazione dell'AFLED, Associazione familiari lavoratori eternit deceduti, promossa dalla Camera del Lavoro e dai protagonisti di questi anni di lotta e rivendicazioni a partire dall'interno delle mura

\footnotetext{
${ }^{25}$ Altopiedi R., "Azione collettiva e costruzione della vittimizzazione. Il caso Eternit", in Studi sulla questione criminale, 1/2013, pp. 31-59.
}

della fabbrica. Nello stesso anno, però, si ammala Piercarlo Busto, ragazzo di 33 anni, sportivo, che conduceva uno stile di vita sanissimo. Un cittadino comune, un bancario che mai aveva avuto contatti con la fabbrica. Nessuno della sua famiglia aveva lavorato alla Eternit e viveva lontano dalla zona. Se può ammalarsi questo ragazzo, il mesotelioma può colpire tutti. La famiglia del giovane volle denunciare l'accaduto e nel manifesto di morte scrisse "per causa dell'amianto è morto Piercarlo Busto". Questo gesto fu importante per il diffondersi della consapevolezza sulle potenzialità nocive dell'amianto. Attirò stampa e televisioni nazionali. La famiglia Busto decide così non solo di denunciare la cosa pubblicamente, ma di attivarsi per ottenere risultati. Nel 1989 fonda l'AEA, associazione esposti amianto, composta di cittadini sensibili alla tematica dell'amianto che, con un impegno costante, preciso e attivo, consente di scuotere le coscienze e di far ottenere i primi risultati, come la messa al bando dell'amianto nel 1992.

Non basta questo risultato a fermare le associazioni di cittadini ed ex lavoratori, che si uniscono in AFEVA, associazioni familiari e vittime amianto, per ottenere giustizia non in senso meramente risarcitorio, ma per diventare il precedente, per impedire il ripetersi di stragi di questo tipo. Infatti, anche se l'azienda non è più attiva, rimane mortalmente pericolosa.

Centrale per il proseguire della vicenda è il caso di un lavoratore di Orbassano che aveva contratto il mesotelioma in uno stabilimento svizzero ed era venuto a morire in Italia; fu questa storia a convincere Raffaele Guariniello, magistrato della procura di Torino, che vi fosse un legame tra i diversi stabilimenti facenti capo 
al gruppo Eternit. Grazie a questa convinzione, vennero estese le indagini a tutti i vertici della multinazionale e Guariniello decise di perseguire non solo gli amministratori italiani, ma anche i massimi dirigenti, cominciando un complesso lavoro di indagine.

\section{Lo storico processo Eternit ${ }^{26}$.}

Il 6 Aprile 2009 inizia il processo Eternit e si conclude con il primo grado di giudizio il 13 Febbraio 2012, giorno in cui entrambi gli imputati De Cartier De Marchienne Louis e Schmidheiny Stephan vengono condannati per i reati contestati. A rendere "storico" il processo, oltre agli impressionanti numeri (66 udienze, 63 testimoni ascoltati, 42 consulenti tecnici di parte, 2897 parti offese e 6392 soggetti costituitisi come parte civile), hanno contribuito i capi di imputazione. Infatti i pubblici ministeri Raffaele Guariniello, Sara Panelli e Gianfranco Colace hanno contestato i reati di cui agli articoli $43727 \mathrm{e}$ $434^{28}$ del Codice Penale. La dimensione del

\footnotetext{
${ }^{26}$ Questo capitolo è nato anche grazie al confronto, avvenuto il 10 Ottobre 2014 a Torino, con Rosalba Altopiedi: ricercatrice dell'università di Torino e consulente del PM Guariniello durante lo storico processo.

${ }^{27}$ Codice Penale, Libro II, Titolo VI-dei delitti contro l'incolumità pubblica, Capo I- dei delitti di comune pericolo mediante violenza, art. 437 "Rimozione od omissione dolosa di cautele contro infortuni sul lavoro", in base al quale: Chiunque omette di collocare impianti, apparecchiature o segnali destinati a prevenire disastri o infortuni sul lavoro, ovvero li rimuove o li danneggia, è punito con la reclusione da sei mesi a cinque anni.

${ }^{28}$ Codice Penale, Libro II, Titolo VI-dei delitti contro l'incolumità pubblica, Capo I- dei delitti di comune pericolo mediante violenza, art. 434 "Crollo di costruzioni o altri disastri dolosi", in base al quale: Chiunque, fuori dei casi preveduti dagli articoli precedenti, commette un fatto diretto a cagionare il crollo di una costruzione o di una parte di essa ovvero un altro disastro è punito, se dal fatto deriva pericolo per la pubblica incolumità, con la reclusione da uno a cinque anni.
}

disastro è uno degli elementi che rende storico il processo; solitamente la pubblica accusa incardina $\mathrm{i}$ procedimenti per esposizione da amianto (o da altra sostanza tossica industriale) sui reati di omicidio (generalmente colposo) e lesioni personali. Questa volta si è scelto di non attribuire rilievo penale ad una miriade di singoli eventi, anche perché il numero di vittime era davvero impressionante e avrebbe impedito tempi rapidi di progressione. La scelta di un accertamento impersonale giustifica le figure di reato: non delitti contro la persona, ma delitti contro l'incolumità pubblica, più idonei ad intercettare il danno alla popolazione. La decisione di non considerare il nome e il cognome di ogni singola vittima (riportati nella sentenza) non deve essere interpretata, a mio avviso, come una volontà di sminuire l'importanza di ogni singola storia, ma piuttosto come il tentativo di rendere chiara la massiva dannosità di questo materiale, di questo evento, di queste scelte, che impattano indifferenziatamente il bersaglio da colpire. I discorsi sugli eventi lesivi e sulla causalità sono stati spostati sul piano collettivo, paragonando, nella sentenza d'appello, gli effetti dello stabilimento ad un fenomeno epidemico, ancora in ascesa.

\section{Il 13 Febbraio 2012 Louis De Cartier e Stephan} Schmidheiny, i due proprietari della multinazionale dell'amianto, sono stati condannati a 16 anni per disastro ambientale doloso e omissione dolosa di cautele antinfortunistiche.Nel Giugno del 2013 si conclude anche il processo di secondo grado: dopo la morte del barone belga Cartier,

La pena è della reclusione da tre a dodici anni se il 
Schmidheiny rimane l'unico imputato, condannato a 18 anni di carcere, due in più della sentenza di primo grado. Sono stati poi stanziati circa 90 milioni di euro destinati al comune di Casale Monferrato e 30 mila euro agli ammalati di patologie legate all'amianto e ai familiari delle vittime. Questo risultato permette ai casalesi di tirare un sospiro di sollievo, soprattutto dopo i tentativi dello svizzero Schmidheiny di venire a patti privatamente con il comune di Casale Monferrato, offrendo il doppio di quanto avesse chiesto, in cambio dell'estromissione dai banchi delle parti civili.

Poi il 19 Novembre 2014 quelle parole, in un processo durato poche ore: "si annulla senza rinvio la sentenza impugnata nei confronti dell'imputato Schmidheiny Stephan Ernst (...) perché il reato è estinto per prescrizione maturata anteriormente alla sentenza di primo grado". E' il dispositivo tombale con cui la Cassazione scrive la parola fine sullo storico processo penale.

Le motivazioni chiariscono come, ad avviso dei giudici di legittimità, il processo Eternit fosse cominciato quando il reato di disastro innominato, disciplinato dall'articolo 434 del Codice Penale, era già prescritto; una sorta di falsa partenza.

La Cassazione ha deciso che nel caso in esame la consumazione del reato non può considerarsi protratta oltre il momento in cui cessarono le emissioni delle polveri e dei residui della lavorazione dell'amianto prodotto dagli stabilimenti, quindi non può considerarsi oltre il Giugno dell'anno 1986, con il fallimento della società. Il termine di prescrizione del delitto, pari al massimo a 15 anni, iniziato a decorrere nel

crollo o il disastro avviene.
1986, era abbondantemente spirato già prima del 2012, motivo per il quale questa decisione porta all'estinzione di tutte le statuizioni civili a favore delle parti offese. La tesi accusatoria avanzata era stata di qualificare il disastro innominato alla stregua di un reato permanente ${ }^{29}$, ritenendo che l'offesa fosse rappresentata dalla perdurante situazione di inquinamento, ancora pericoloso per l'incolumità pubblica, e che la condotta degli imputati di omesso impedimento del protrarsi del disastro fosse tuttora in corso. Tutto questo consentiva di "schivare" la prescrizione, affermando che il relativo termine non avesse nemmeno iniziato a decorrere. Impostazione respinta dalla corte di Cassazione perché la norma in questione non prevede l'obbligo di far cessare la situazione antigiuridica prodotta, quindi non prevede l'obbligo per l'imputato di risolvere i drammatici effetti causati dalla propria società. Inoltre, aggiunge la Cassazione, se tale obbligo secondario fosse concepibile, dovrebbe essere previsto per ogni fattispecie che non contempli la distruzione del bene protetto, qualificando, così, come "permanenti" reati istantanei come il furto, ad esempio.

Nonostante l'ingegnosa e innovativa ricostruzione, la Cassazione non deraglia dai binari dell'interpretazione consolidata nella dottrina.

Il meticoloso lavoro dei PM e dei loro collaboratori poteva portare al precedente, aveva acceso l'attenzione della stampa, anche di quella internazionale, $\mathrm{ma}$ si è scontrato con la tradizione giurisprudenziale del nostro paese che

${ }^{29}$ E' permanente il reato le cui conseguenze si protraggono nel tempo. Il perdurare della lesione dipende esclusivamente dalla volontà dell'agente. Un classico esempio di reato permanente è il "sequestro di persona". 
non porta le norme a plasmarsi al caso specifico, ma, più spesso, cerca di incastrare il caso in esame in norme pre-esistenti, inflessibili. Creare il precedente non è stato strutturalmente possibile.

In una tradizione di civil law come la nostra, il diritto penale è fedele alla legge: il diritto non può piegarsi alla giustizia sostanziale, perché ciò che sembra giusto oggi può non esserlo domani $^{30}$. Come ha concluso la requisitoria davanti alla Cassazione il Procuratore Generale Iacovello: "ci sono dei momenti in cui diritto e giustizia vanno da parti opposte; ’̀ naturale che le parti offese scelgano la strada della giustizia, ma quando il giudice è posto di fronte alla scelta drammatica tra diritto $e$ giustizia non ha alternativa. Un giudice sottoposto alla legge, tra diritto e giustizia deve scegliere il diritto'’1.

\section{Associazionismo e riconoscimento ${ }^{32}$.}

I movimenti, dal verbo latino moveo, che significa sì, muovere, ma anche mutare, trasformare, hanno lo scopo di generare un cambiamento, a partire dal basso, a partire, in casi come questo, dalle stesse vittime, al fine di colmare un vuoto istituzionale, che rende le vittime le grandi trascurate della nostra società e, quindi, doppiamente vittimizzate.A favorire la mobilitazione per ottenere un cambiamento

\footnotetext{
${ }^{30}$ Panebianco A., Il potere, lo Stato, la libertà, il Mulino, Bologna, 2004.

${ }^{31}$ Schema della requisitoria pronunciata avanti alla Sezione I penale della Corte di Cassazione nel processo Eternit - 19 novembre 2014 dal Cons. Francesco Iacoviello Sostituto Procuratore Generale presso la Corte di Cassazione pubblicati da Diritto Penale Contemporaneo, consultabile in www.penalecontemporaneo.it.

${ }^{32}$ Le modalità di reazione sono state estrapolate dalle interviste che ho condotto a Bruno Pesce, Nicola Pondrano, Romana Blasotti e Luca Cavallero il 24 Settembre 2014 e il 27 Gennaio 2015.
}

necessario al bene comune pare essere l'interazione. I singoli partecipano, agiscono e reagiscono grazie a chi lo ha già fatto e, nella storia di Casale Monferrato, l'interdipendenza ha giocato un ruolo fondamentale ${ }^{33}$.

L'associazionismo può essere considerato una forma moderna di elaborazione del lutto: il gruppo ripara ciò che il singolo faticherebbe a riparare da solo.

In una società come la nostra è difficile per le vittime ottenere il riconoscimento e la tutela necessarie a superare la propria condizione e a ottenere giustizia, sia sul piano informale, che sul piano istituzionale; soprattutto per i comportamenti illeciti perpetrati dalle imprese, per le caratteristiche strutturali di queste azioni, analizzate in precedenza.

Un tentativo di definizione, riconoscimento ed ottenimento di giustizia è stato portato avanti dalle associazioni attive nel territorio di Casale Monferrato. L'associazione storica attiva nel territorio è l'Afeva, Associazione dei familiari delle vittime dell'amianto, siano essi lavoratori o cittadini.

Dopo l'ottenimento, nel 1992, della messa a bando dell'amianto, l'associazione ottiene visibilità nazionale e internazionale e non interrompe le proprie attività. Promuove attività di sensibilizzazione e di educazione alla cittadinanza, coinvolge le scuole nella costruzione di una consapevolezza e di una memoria comune, per trasmettere a una generazione che non ha visto, in prima persona, Casale Monferrato ricoperta da una patina di polvere, il dramma che ha segnato tante famiglie.

\footnotetext{
${ }^{33}$ Altopiedi R., "Azione collettiva e costruzione della vittimizzazione. Il caso Eternit", in Op. cit.
} 
I primi attivisti, che per lo più provenivano dal movimento sindacale, tra questi Bruno Pesce e Nicola Pondrano, hanno saputo coinvolgere un numero sempre crescente di persone svincolate dai partiti. Tra queste Romana Blasotti, "La Romana": una donna arrabbiata, che conosce bene gli effetti collaterali dell'amianto. Romana Blasotti non rappresentava un partito, un sindacato, ma le persone normali e in situazioni come questa le istituzioni rischiano di delegittimare una lotta, soprattutto in una situazione storica di grande sfiducia nei loro confronti, come quella contemporanea. "Se lo fa lei lo posso fare anche io", questo quello che viene da pensare, guardando e conoscendo questa signora che, dopo la sentenza della Cassazione, ha deciso di dimettersi dal ruolo di presidente dell'associazione.

Ai soggetti collettivi che hanno fermentato il territorio di Casale Monferrato va il merito di aver smascherato la natura criminale dello stabilimento Eternit e di aver contribuito alla costruzione di un'identità collettiva. Nelle interviste condotte durante lo studio di caso, gli altolocati dirigenti dell'Eternit vengono chiamati, a più riprese, criminali, a voler attribuire le giuste etichette.

Le vicende private sono divenute pubbliche e l'associazione è diventata attore collettivo, con propri interessi: un gruppo di pressione che vuole interagire con le istituzioni, con i rappresentanti dello stato, che va oltre il livello di supporto e affetto presente in altre associazioni ${ }^{34}$. Grazie, poi, ad un minuzioso lavoro di raccolta di documenti, di ascolto e supporto, l'Afeva ha dato un nome e una dignità

\footnotetext{
${ }^{34}$ Altopiedi R., Op cit.
}

alle oltre 3.000 vittime che lo stabilimento Eternit ha causato a Casale Monferrato.

Nonostante l'indiscutibile indispensabilità di questo movimento, sono anche emersi dei piccoli punti critici nei confronti dell'operato dell'Afeva, sia da parte di Romana Blasotti, sia da parte di Luca Cavallero, uno dei fondatori dell'altra associazione attiva nel territorio di Casale Monferrato, Voci della Memoria. È l'ormai ex presidentessa dell'Afeva che, nella intervista svolta dopo la drammatica sentenza di Cassazione, afferma:"C'era l'antagonista, la battaglia da combattere e le energie sono andate lì, ma in alcuni momenti avremmo potuto investire su persone capaci.Molti giovani in gamba e svegli si sono avvicinati a noi e l'Afeva non li ha coltivati a sufficienza".

Tra i giovani che non sono stati coltivati a sufficienza Luca Cavallero: intelligente, impegnato e appassionato. Assieme ad altri ragazzi "stanchi di essere trattati da giovani”, registrano nel 2010 una nuova associazione: Voci della Memoria. Ognuno dei membri è una voce, una voce diversa, che va raccontata per fare memoria, non in senso commemorativo o celebrativo, ma per fare in modo che dal passato e dal vissuto si possa apprendere. Ogni membro ha una storia diversa, quella di "Luca di Voci" è la storia di Casale Monferrato. La storia di Casale Monferrato è la storia di tutti, "non solo dell'Afeva" e, grazie a Voci, viene raccontata anche al di fuori di canali istituzionali. Rimane comunque a stretto contatto con l'operato dell'Afeva, con questa comunica e collabora, ma si definisce il suo braccio armato. Nonostante le differenze operative, entrambe le associazioni si definiscono a-partitiche, ma politiche. Entrambe hanno un solo scopo: raccontare, farsi sentire, 
impedire che quanto accaduto ieri possa ripetersi domani. Queste le parole "della Romana": "Mi auguro che $i$ giovani che saranno gli adulti di domani avranno più sicurezza nel portare avanti la lotta. Il prossimo non è carne da macello. Non si guadagna dalla morte degli altri. Non si può restare indifferenti. Questa è stata una strage".

\section{Conclusioni.}

La storia di Casale Monferrato è la storia di una battaglia di civiltà. Una battaglia per la giustizia sociale; non ha colore. A generare la forza di aggregazione è il coinvolgimento di tutti.

L'Italia è una Repubblica democratica fondata sul lavoro e questa storia racconta come proprio il lavoro abbia ucciso migliaia di persone. Questa è un'ingiustizia, ancora drammaticamente presente in diverse realtà del nostro Paese e per la quale ancora manca una dignitosa tutela normativa.

E' giusto lavorare per morire? E' giusto doversi trovare a scegliere tra salute e lavoro? E, ancora, è giusto che un'impresa avveleni un territorio per soddisfare interessi di profitto?

La risposta è una sola: no.

Il fatto che questo stia ancora accadendo dovrebbe farci riflettere su quanto sia necessario diffondere una cultura sociale, un'idea di impresa responsabile, consapevole di agire all'interno di un territorio del quale non può fare a meno e che, per questo ha l'obbligo, anche morale, di tutelare. Territorio che va tutelato come è necessario tutelare la salute dei lavoratori, anch'essi indispensabili per il funzionamento dell'impresa, con un nome, un cognome, una famiglia.

E' necessario formare e informare.
Casale Monferrato ha intrapreso un percorso che testimonia che, di fronte alla scelta che spesso viene posta tra lavoro e salute, o, ancora, tra lavoro e ambiente, non si può rispondere: è un trade-off che non deve più esistere.

Occorre un percorso di diffusione e condivisione di responsabilità che coinvolga Stato, imprese e cittadini, che spesso sono i primi a pagare il prezzo di scelte irresponsabili e che, se riescono ad apprendere la potenzialità del loro ruolo all'interno del contesto sociale, si rivelano un'indispensabile strumento di pressione per generare un cambiamento.

\section{Bibliografia.}

- Altopiedi R., Un caso di criminalità di impresa. L'Eternit di Casale Monferrato, L'Harmattan Italia, Torino, 2011.

- Altopiedi R., "Azione collettiva e costruzione della vittimizzazione. Il caso Eternit”, in Studi sulla questione criminale, n. 1/2013, pp. 31-59.

- Altopiedi R., Panelli S., "Il grande processo", in Quaderno di storia contemporanea ISRAL, n. 51/2012, pp. 17-77.

- Balloni A., Viano E. (a cura di), IV Congresso Mondiale di Vittimologia. Atti della giornata bolognese, Clueb, Bologna, 1989.

- Bravo F., Criminalità economica e controllo sociale. Impresa etica e responsabilità ex d.lgs. 231/01, Clueb, Bologna, 2010.

- Bravo F., "Criminalità economica 'violenta', compliance models e rating di legalità delle imprese", in Balloni A., Bisi R., Sette R., Manuale di Criminologia vol. II, Clueb, Bologna, 2013.

- Carnevale F., Chellin E., Amianto. Miracoli, virtù, viæi, Tosca, Firenze, 1992.

- Carnevale F., "Amianto: miracoli, virtù e vizi del passato e del presente", in Maggi B. e Rulli G. (a cura di), Quale insegnamento dall' "epopea" dell'amianto?, Tao Digital Library, Bologna, 2014.

- Cottino A., Disonesto ma non Criminale. La giustizia e i privilegi di potenti, Carocci, Roma, 2005. 
- Chambliss W., "Policing the Ghetto Underclass: the politics of law and law enforcement", in Social Problem, n. 41/1994, pp. 177-194.

- Di Amato A., La responsabilità penale da amianto, Giuffrè, Milano, 2003.

- Doll R., "Morality from lungcancer in asbestosworkers", in Brit. J. Industr. Med., n. 12/1955, pp. 81-87.

- Douglas M., Rischio e colpa, il Mulino, Bologna 1996.

- Elias R., "Which victim movement? The politics of victim Policy", in Fattah E.A. (a cura di), Towards a Critical Victimology, St. Martin's Press, New York, 1993.

- Friedberg E., Il potere e la regola. Dinamiche dell'azione organizzata, Etas, Milano, 1994.

- Gallino L., Dizionario di Sociologia, Utet, Torino, 1978.

- Gallino L., L'impresa irresponsabile, Einaudi, Torino, 2009.

- Galluccio L., Gavino P., Responsabilità penale e amministrativa delle imprese, Gruppo 24Ore, Milano, 2009.

- Hart H. L. A., Punishment and Responsability, Oxford University Press, New York, 1968.

- Martucci P., La criminalità economica. Una guida per capire, Laterza, Roma-Bari, 2006.

- Misztail B. A., Trust in modern society, Polity Press, Cambridge, 1996.

- Mosconi G., Padovan D., La fabbrica dei delinquenti. Processo penale e meccanismi sociali di costruzione del condannato, L'Harmattan Italia, Torino, 2005.

- Sutherland E.H., Il crimine dei colletti bianchi. La versione integrale, Giuffrè, Milano, 1987.

- Sutherland E.H., "Is 'white-collar crime' a crime?", in American SociologicalReview, n. 2/1944, pp. 132-139.

- WHO, World report on violence and beath, Geneva, 2002.

- Young M., Stain J., The history of the crime victims' movement in the United State, U.S. Government Printing Office, Washington D.C., 2005.

- Zamperini A., Psicologia sociale delle responsabilità, UTET, Torino, 1998.

- Zagrebelsky V., "L'organizzazione del lavoro: esame delle notizie di reato, i flussi di lavoro e le sezioni specializzate", in Quaderni del Consiglio Superiore, n. 78/1995, pp. 17-44. 